\title{
Identification of Three Particular Morphological Phenotypes in Sporadic Thoracic Aortic Aneurysm: Phenotype III As Sporadic Thoracic Aortic Aneurysm Biomarker in Aged Individuals
}

\author{
Carmela Rita Balistreri, ${ }^{1, *}$ Emiliano Maresi, ${ }^{2, *}$ Calogera Pisano, ${ }^{3}$ Federica Maria Di Maggio, ${ }^{1}$ \\ Loredana Vaccarino, Calogero Caruso, Domenico Lio, ${ }^{1}$ Giovanni Ruvolo, ${ }^{3}$ Giuseppina Candore ${ }^{1}$
}

\begin{abstract}
Aging has a striking impact on the heart and the vascular system, particularly on the large elastic arteries (i.e., aorta), resulting in a multitude of changes at different structural and functional levels. As result, medial degeneration (MD) occurs. A characteristic example of MD is sporadic thoracic aortic aneurysm (S-TAA), whose patho-physiological mechanisms remain unclear. In this study, typical MD morphological phenotypes were researched in S-TAA cases and control aorta specimens by histopathological and immunohistochemical analyses. Three phenotypes (I, II, and III) were detected, but mainly the phenotype III was observed. Elevated cystic MD, plurifocal medial apoptosis, and increased metalloproteinase- 9 amount characterize it. In addition, it was significantly correlated with the severity of elastic fragmentation, hypertension, and smoking, and particularly with advancing age. Thus, phenotype III might represent the typical MD phenotype associated with S-TAA in old people that have a major risk of aorta rupture and dissection independently on aneurysm diameter. This might permit the assumption that phenotype III with its typical histological abnormalities is an optimal biomarker of rupture and/or dissection in aged individuals and is useful both for applying different surgical approaches and providing appropriate surgical indications.
\end{abstract}

\section{Introduction}

A GING HAS A STRIKING IMPACT on heart and vascular system, particularly on the large elastic arteries (i.e., aorta), determining a multitude of changes at different structural and functional levels. ${ }^{1-3}$ As result, medial degeneration (MD) occurs. ${ }^{4}$ At the macroscopic level, this pathological entity induces weakening of the aorta wall and a progressive stiffening. ${ }^{4,5}$ At the microscopic level, MD is characterized by endothelial dysfunction, increased oxidative stress, inflammatory reaction, inflammatory cell infiltration in the aortic wall, and apoptosis of vascular smooth muscle cells (VSMCs), followed by degeneration of aortic media, elastin fracture, and degradation. ${ }^{4,5}$ In turn, this results in aortic dilatation and aneurysm, and an increased risk of the onset of complications, i.e., aortic dissection and rupture. ${ }^{4}$ A characteristic model of $\mathrm{MD}$ is sporadic thoracic aortic aneurysm (S-TAA). ${ }^{4}$ This is becoming a serious health risk because of growing enhancement of underlying diseases, i.e., hypertension and aging, in Western populations. $^{6-10}$ The aged population seems to experience a increased incidence of S-TAA with advancing of years, as recently reported by epidemiological studies performed in geographic regions with stable populations with little out- or in-migration, such as in Minnesota and Sweden. ${ }^{9,10} \mathrm{Hy}-$ pertension is a widely prevalent and important risk factor for cardiovascular diseases, including S-TAA. ${ }^{6}$ On the other hand, new guidelines have emphasized hypertension as the commonest cause of preventable death and as being significantly increased in aged population of developed countries. $^{6}$

In contrast to the extensive study of syndromic and familial TAAs, the elucidation on the genetic, molecular, and cellular mechanisms of sporadic TAA forms has recently begun. ${ }^{4}$ On the basis of these observations, the eventual presence of typical MD morphological phenotypes was

\footnotetext{
${ }^{1}$ Immunosenescence Group, Department of Department of Patho-biology and Medical and Forensic Biotechnologies, ${ }^{2}$ Department of Pathologic Anatomy, and ${ }^{3}$ Unit of Cardiac Surgery, Department of Surgery and Oncology, University of Palermo, Palermo Italy.

*These authors contributed equally to this study.
} 
studied in aorta sporadic TAA samples through histopathological and immunohistochemical analyses. Our major goal was to identify one phenotype associated mainly with the risk of aorta rupture and dissection in old people.

\section{Materials and Methods}

Aorta specimens from 100 S-TAA patients $(97$ men and 13 women, whose median age was $62.95 \pm 11.44$ years) and 30 control individuals $(20$ men and 10 women, mean age $62.9 \pm 11.57)$ died of causes unrelated to aortic disease and no sepsis, confirmed by autopsy, were collected. To perform histopathological and immunohistochemical analyses, patient and control aortas were fixed in $10 \%$ neutral buffered formalin for $24 \mathrm{hr}$, and then processed for routine paraffin embedding. For examinations by microscopy, multiple histological sections from each sample were prepared and stained with Hematoxylin \& Eosin, Weigert-van Gieson, and Alcian-Periodic Acid Schiff (PAS). TUNEL (terminal deoxynucleotidyl transferase-mediated dUTP nick endlabeling) testing and immuno-histochemical assessment for the evaluation of metalloproteinase-9 (MMP-9) levels and medial apoptosis were also performed, using procedures described in Supplementary Data (Supplementary Data are available at www.liebertonline.com/rej/).

The aortic wall was mainly evaluated for following histological features: (1) Fibrosis (defined as an increase in interstitial collagen); (2) medio-necrosis (defined as a focal loss of smooth muscle cell nuclei in the media); (3) cystic medial necrosis (defined as mucoid material accumulation); (4) focal or medial plurifocal apoptosis; (5) elastic fragmentation (defined as focal fragmentation of elastic lamellae

SF1 SF2 in the media); (6) amounts of MMP-9 (see Figs. S1 and S2).

Histo-pathological abnormalities of aortic wall were graded and defined according to the definitions and grading systems used by Matthias Bechtel and colleagues ${ }^{11}$ and also described in our recent studies (see Figs. S1 and S2). They were defined as follows. Cystic medial change: Grade I, minute cystic cavities holding a basophilic ground substance that occupy the total width of one lamellar unit; grade II, cystic cavities holding a basophilic ground substance that occupy the total width of two or more contiguous lamellar units; and grade III, large cystic cavities, holding a basophilic ground substance, that occupy the total width of aortic media (Fig. S1). Elastic fragmentation: Grade I, up to five foci of elastic fibers interruption in two-four neighboring lamellar units in one microscopic field of $100 \times$ magnification; grade II, more than five foci of elastic fibers interruption in two-four neighboring lamellar units; grade III, elastic fibers interruptions occupy the total width of aortic media with disarray of smooth muscle cells (Fig. S1). Fibrosis: Grade I, an increase of collagen fibers in an area less than one-third of the total medial thickness; grade II, an increase of collagen fibers occupying between one-third and two-thirds of the medial thickness; grade III, an increase of collagen fibers that occupy more than two-thirds of the medial thickness (Fig. S1). Medial necrosis: Grade I, focal loss of smooth muscle cells nuclei in an area less than onethird the thickness of the media; grade II, focal loss of smooth muscle cells nuclei that occupy between one-third and two-thirds the medial thickness; grade III, focal loss of smooth muscle cells nuclei that occupy more than two-thirds the medial thickness (Fig. S1). Atherosclerotic aneurysms: We have defined atherosclerotic aneurysms as those that showed macroscopic intimal calcific plaques or microscopic intimal fibrotic thickness that is one-third that of the aortic media thickness.

\section{Statistical analysis}

Correlations were assessed using Spearman rank correlation. A $p<0.05$ was considered statistically significant.

\section{Results}

Interestingly, 73 case aorta tissues were with the typical MD and without atherosclerotic lesions. In these tissues, three phenotypes (I, II, and III) were detected having a different quantitative relationship of cystic MD, fibrosis, apoptosis, and amount of MMP-9 (see Figs. S1 and S2). They were described as follows: Phenotype I, cystic medial degeneration balanced by a substitutive fibrosis, in absence of medial apoptosis and with a low MMP-9 concentration; phenotype II, higher cystic medial degeneration than substitutive fibrosis, with focal medial apoptosis, and with mainly a modest MMP-9 amount; phenotype III, elevated cystic medial degeneration, without substitutive fibrosis, with plurifocal medial apoptosis, and with an elevated MMP-9 concentration.

Among these, phenotype III was mainly (63 vs. 73) observed in case aortas. It showed elevated cystic MD, plurifocal medial apoptosis, and increased MMP-9 amount (see Fig. 1A,B). In addition, phenotype III was significantly correlated with the severity of elastic fragmentation, hypertension, smoking, and particularly with advancing age $(r=0.497$, $p=0.0001 ; r=0.267, p=0.03 ; r=0.342, p=0.006 ; r=0.567$, $p=0.0001$, respectively; by non-parametrical Spearman correlation test; data not shown). In contrast, no correlation was observed with aorta diameter (data not shown). Thus, phenotype III might represent the typical MD phenotype associated with major risk of aorta rupture and dissection independently on aneurysm diameter.

\section{Discussion}

Aortic disease is currently a large health concern because it is common particularly in advancing age and can lead to fatal outcomes. ${ }^{12}$ A variety of conditions affect the aorta with progressing years; the most common are aneurysm, dissection, occlusion owing to atherosclerosis, and general stiffening. ${ }^{12}$ These conditions are characterized by microstructural changes that frequently are the result of vascular aging. ${ }^{1-3}$ Indeed, aging determines the loss of molecular fidelity at cellular, tissue, and organ levels, including the heart and vascular system, followed by a progressive entropy, which renders patients more easily vulnerable to internal and external stressors, frailty, disability, and disease. ${ }^{13}$ In the thoracic aorta, age-related modifications give rise to a pathological entity, $\mathrm{MD}$, having catalyst and accelerator effects for the onset of S-TAA. ${ }^{4}$ Thus, S-TAA risk increases with biological aging. Little has been known until now about the histopathological MD-related phenotypes associated with a major risk for S-TAA and its complications, especially in old people. ${ }^{4,14-16}$ This led us to perform histopathological studies to identify phenotypes of 

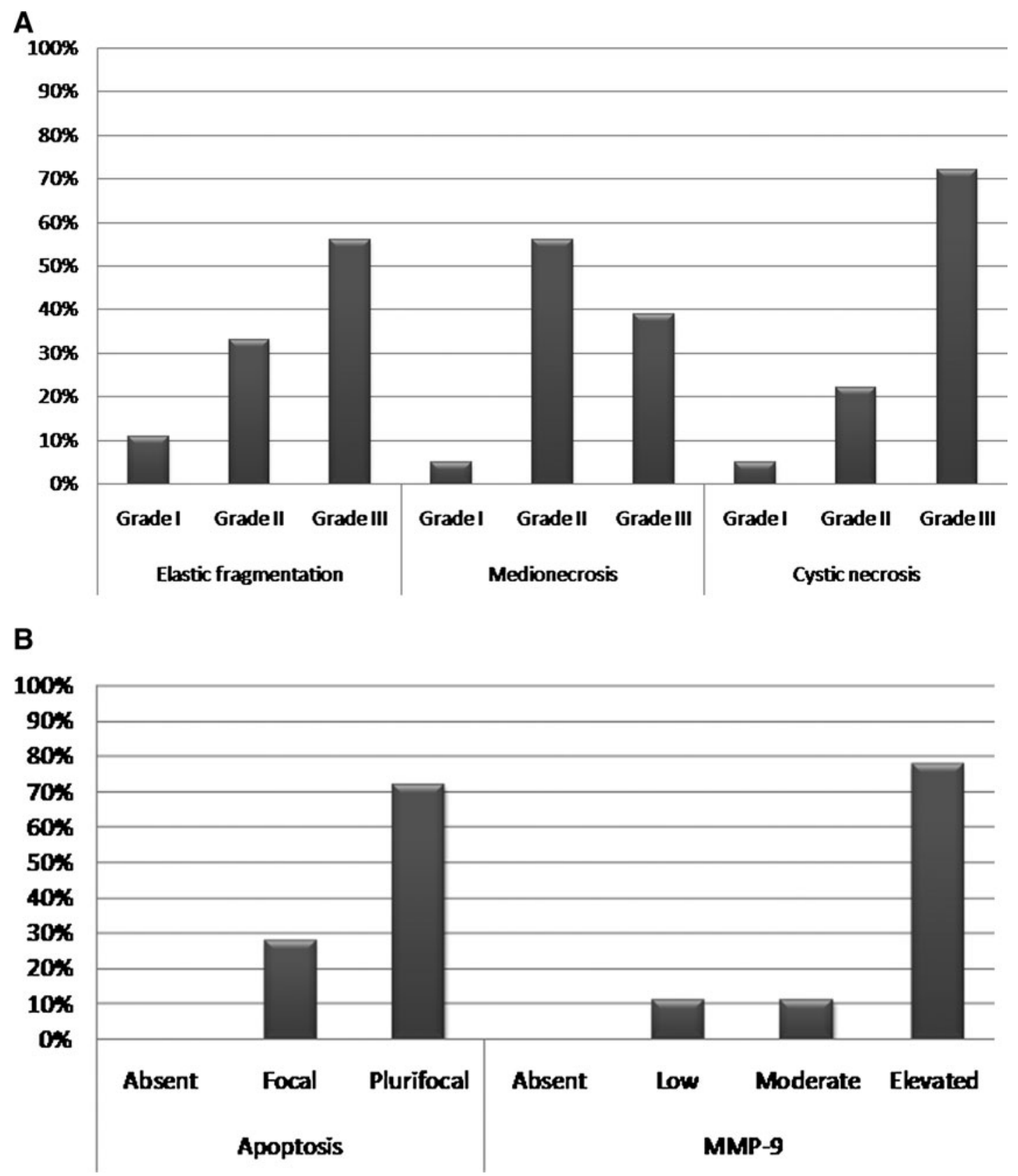

FIG. 1. (A and B) Aorta wall abnormalities characterizing phenotype III. (A) Elastic fragmentation, medio-necrosis, and cystic necrosis essentially of grades II and III were found. (B) Focal and plurifocal apoptosis were also found in all tissue aorta samples from patients versus controls. In addition, low, moderate, and elevated metalloproteinase-9 (MMP-9) amounts were also observed.

S-TAA, which, more than others, evolve into dissection or rupture. ${ }^{17,18}$

In a previous study, we detected a morphological identity of medial lesions that might be assumed as precursor and consequently as optimal biomarker of type A dissection (TAD), independently of aneurysm diameter or valvular disorder. ${ }^{17}$ On the other hand, we observed that the severity of aortic media degeneration in TAD cases is not related to the diameter of the aneurysm. ${ }^{17}$ Other research groups frequently found the onset of TAD in patients with a normal aortic diameter. ${ }^{17,18}$ These promising findings encouraged us to find phenotypes associated with the risk of aorta rupture and dissection in aged S-TAA individuals. Thus, in the present study, histopathological and immunohistochemical analyses were assessed in aorta specimens from 100 S-TAA patients with median age of $62.95 \pm 11.44$ years and ageand gender-matched controls. Interestingly, we observed that a large number of aorta samples showed a morphological identity, defined as phenotype III and characterized by elevated cystic MD, plurifocal medial apoptosis, and increased MMP-9 levels. This phenotype appears to be significantly correlated with the severity of elastic fragmentation. In addition, it shows a relative absence of a reparative fibrosis, and consequently it might mainly predispose the 
S-TAA patients to aortic rupture. These data seem to be in agreement with the emerging literature data suggesting the key involvement of an up-regulation of metalloproteinase and apoptosis correlated with inflammatory age-related processes and genetic factors in the pathophysiology of S-TAA. ${ }^{18}$ On the other hand, we recently suggested that inflammation producing MMPs, cytokines, and death mediators seem to be the shared pathological mechanism for TAD. In addition, significant associations between single-nucleotide polymorphisms in inflammatory genes and the TAD risk were also reported in our study. ${ }^{18}$

Furthermore, phenotype III seems to be the result of three major risk factors of S-TAA, including hypertension, smoking, and advancing age. Indeed, a significant positive correlation was, observed between phenotype III and these risk factors. On the other hand, in a recent study we demonstrated that smoking, hypertension, and age were the exclusive risk S-TAA factors significantly associated with the reduction of the mean leukocyte telomere length. ${ }^{19}$ Their association seems plausible with the biological effects that they mediate on the aortic wall. In contrast, no correlation was observed with aortic diameter.

Together our results, although obtained from a relatively large number of individuals, might indicate that phenotype III, with its typical histological abnormalities, is an optimal biomarker of rupture and/or dissection in aged individuals and would be useful both for applying different surgical approaches and providing appropriate surgical indications. On the other hand, an increase of S-TAA incidence in the aged population has been recently observed by epidemiological studies. ${ }^{7,9,10}$ In addition, this might be useful because S-TAA is clinically and predominantly a silent ailment until rupture or dissection occurs and is insidious in its onset and progression. Furthermore, until now its diagnosis is also exclusively based on imaging technologies, and no blood tests exist. Certainly, our findings require continued research on the genetic, cellular, and molecular mechanisms involved in the development of this phenotype. It and combined efforts might lead to further elucidation about the role of S-TAA phenotype III, especially in old people.

A major advance would be to be translate these findings into individualized and effective pharmacological treatments oriented toward molecular and genetic mechanisms, allowing for tailored medical and surgical approaches to this very serious condition. ${ }^{20}$ Accordingly, recently Castellano and colleagues emphasized that we are ignoring this disease, which is growing in incidence and characterized by a different location and etiology with respect to abdominal aorta aneurysm (AAA). These aortic diseases need to be considered as distinct entities and not hastily grouped together. Thus, the aortic diameter and therapies used for AAA care are not suitable for S-TAA, as recently evidenced by new guidelines for S-TAA. ${ }^{21}$ Clinical trials for S-TAA are required before medical therapies, such as $\beta$-blockers, angiotensin-converting enzyme inhibitors, angiotensin receptor blockers, statins, or macrolide antibiotics, can be recommended. On the other hand, we have proposed in our recent studies that a surgical approach for patients with $\mathrm{S}$ TAA should consider not only the diameter of the aortic aneurysm portion but also the histological features and the genetic risk profile. ${ }^{17,18,22}$

\section{Acknowledgment}

This work was supported by grants from the Italian Ministry of Education, University and Research to C.C.

\section{Author Disclosure Statement}

No competing financial interests exist.

\section{References}

1. Karavidas A, Lazaros G, Tsiachris D, Pyrgakis V. Aging and the cardiovascular system. Hellenic J Cardiol 2010; 51:421-427.

2. Laurent S. Defining vascular aging and cardiovascular risk. J Hypertens. 2012;30(Suppl):S3-S8.

3. Bachschmid MM, Schildknecht S, Matsui R, Zee R, Haeussler D, Cohen RA, Pimental D, Loo BV. Vascular aging: Chronic oxidative stress and impairment of redox signaling-consequences for vascular homeostasis and disease. Ann Med 2013;45:17-36.

4. El-Hamamsy I, Yacoub MH. Cellular and molecular mechanisms of thoracic aortic aneurysms. Nat Rev Cardiol 2009;6:771-786.

5. Kovacic JC, Moreno P, Nabel EG, Hachinski V, Fuster V. Cellular senescence, vascular disease, and aging: Part 2 of a 2-part review: Clinical vascular disease in the elderly. Circulation. 2011;123:1900-1910.

6. Aronow WS, Fleg JL, Pepine CJ, et al. ACCF/AHA 2011 Expert Consensus Document on Hypertension in the Elderly: A Report of the American College of Cardiology Foundation Task Force on Clinical Expert Consensus Documents. Circulation 2011;123:2434-2506.

7. Olsson C, Thelin S, Ståhle E, Ekbom A, Granath F. Thoracic aortic aneurysm and dissection: increasing prevalence and improved outcomes reported in a nationwide population-based study of more than 14,000 cases from 1987 to 2002. Circulation 2006;114:2611-2618.

8. Demographic population data. Available at www.istat.it Accessed January 16, 2014

9. Clouse WD, Hallett JW Jr, Schaff HV, Spittell PC, Rowland CM, Ilstrup DM, Melton LJ 3rd. Acute aortic dissection Population-based incidence compared with degenerative aortic aneurysm rupture. Mayo Clin Proc 2004;79:176-180.

10. Acosta S, Ogren M, Bengtsson H, Bergqvist D, Lindblad B, Zdanowski Z. Increasing incidence of ruptured abdominal aortic aneurysm: A population-based study. J Vasc Surg 2006; 44:237-243.

11. Matthias Bechtel JF, Noack F, Sayk F, Erasmi AW, Bartels C, Sievers HH. Histopathological grading of ascending aortic aneurysm: Comparison of patients with bicuspid versus tricuspid aortic valve. J Heart Valve Dis 2003;12: 54-59.

12. Tsamis A, Krawiec JT, Vorp DA. Elastin and collagen fibre microstructure of the human aorta in ageing and disease: A review. J R Soc Interface 2013;10:20121004.

13. Balistreri CR, Candore G, Accardi G, Colonna-Romano G, Lio D. NF- $\kappa$ B pathway activators as potential ageing biomarkers: Targets for new therapeutic strategies. Immun Ageing 2013;10:24

14. Ince $\mathrm{H}$, Nienaber CA. Etiology, pathogenesis and management of thoracic aortic aneurysm. Nat Clin Pract Cardiovasc Med 2007:4:418-427.

15. Elefteriades JA, Farkas EA. Thoracic aortic aneurysm clinically pertinent controversies and uncertainties. J Am Coll Cardiol 2010;55:841-857. 
16. Fernandez-Moure JS, Vykoukal D, Davies MG. Biology of aortic aneurysms and dissections. Methodist Debakey Cardiovasc J 2011;7:2-7.

17. Pisano C, Maresi E, Merlo D, Balistreri CR, Candore G, Caruso M, Codispoti M, Ruvolo G. A particular phenotype of ascending aorta aneurysms as precursor of type A aortic dissection. Interact Cardiovasc Thorac Surg 2012;15: 840-846.

18. Balistreri CR, Pisano C, D'Amico T, Palmeri C, Candore G, Maresi E, Ruvolo G. The role of inflammation in type A aortic dissection: data of a pilot study. Eur J Inflammation 2013; 11:269-278.

19. Balistreri CR, Pisano C, Merlo D, Fattouch K, Caruso M, Incalcaterra E, Colonna-Romano G, Candore G. Is the mean blood leukocyte telomere length a predictor for sporadic thoracic aortic aneurysm? Data from a preliminary study. Rejuvenation Res 2012;15:170-173.

20. Castellano JM, Kovacic JC, Sanz J, Fuster V. Are we ignoring the dilated thoracic aorta? Ann NY Acad Sci 2012; 1254:164-174.

21. Hiratzka LF, Bakris GL, Beckman JA, et al. ACCF/AHA/ AATS/ACR/ASA/SCA/SCAI/SIR/STS/SVM guidelines for the diagnosis and management of patients with Thoracic Aortic Disease: A report of the American College of Cardiology Foundation/American Heart Association Task Force on Practice Guidelines, American Association for
Thoracic Surgery, American College of Radiology, American Stroke Association, Society of Cardiovascular Anesthesiologists, Society for Cardiovascular Angiography and Interventions, Society of Interventional Radiology, Society of Thoracic Surgeons, and Society for Vascular Medicine. J Am Coll Cardiol 2010;55:e27-e129.

22. Pisano C, Maresi E, Balistreri CR, Candore G, Merlo D, Fattouch K, Bianco G, Ruvolo G. Histological and genetic studies in patients with bicuspid aortic valve and ascending aorta complications. Interact Cardiovasc Thorac Surg 2012; 14:300-306.

Address correspondence to: Carmela Rita Balistreri Department of Pathobiology and Medical and Forensic Biotechnologies University of Palermo Corso Tukory 211 Palermo, 90134 Italy

E-mail: carmelarita.balistreri@unipa.it

Received: September 25, 2013 Accepted: September 25, 2013 


\section{Supplementary Data}

\section{Immunohistochemical assays}

Immunohistochemical analyses were performed on 5- $\mu \mathrm{m}$ thick paraffin-embedded sections. Deparaffinized sections were treated for $20 \mathrm{~min}$ in a microwave oven in $10 \mathrm{mM}$ citrate buffer $(\mathrm{pH} 6)$ or Tris/EDTA $(\mathrm{pH} 9)$. Sections were then incubated for $1 \mathrm{hr}$ with specific monoclonal antibodies (mAbs) against metalloproteinase-9 (MMP-9) (clone 15W2, NCL-MMP9 439, Novocastra Laboratories Ltd, UK 1:50) or isotype-matched controls at appropriate dilutions. After washing in $1 \times$ Tris-buffered solution (TBS), staining was performed by biotinylated link antibody and streptovidin labeled with Peroxidase Kits (Dako, North America, Inc., USA) and was detected using 3-amino-9-ethylcarbazole (AEC) substrate chromogen. After counterstaining of cells and tissue sections was performed using aqueous Hematoxylin (Novocastra Laboratories Ldt, UK).

Inflammatory and immune cells were counted in 10 contiguous high-power fields (magnification, $400 \times$ ) under an Olympus fluorescent microscope (America Inc., Melville, $\mathrm{NY}$ ) by two independent observers.

\section{TUNEL testing}

We performed terminal deoxynucleotidyl transferase (TdT)-mediated X-deoxyuridine triphosphate nucleotide (XUTP) nick end-labeling (TUNEL) reaction (In Situ Cell Death Detection Kit, Roche Diagnostics S.p.A, Milano, Italy) on full-thickness aortic wall paraffin sections $(5 \mu \mathrm{m})$. Tissues were deparaffined and then permeablized with phosphatebuffered saline (PBS) $0.1 \%$ sodium citrate/0.1\% Triton X-100. Specimens were then incubated with TdT and fluoresceinlabeled dUTP in a humidified atmosphere for $1 \mathrm{hr}$ at $37^{\circ} \mathrm{C}$. In situ apoptosis staining was revealed by using an AP converter. DNA strand breaks were detected by using the 5-bromo4-chloro-3-indolyl-phosphate/nitro blue tetrazolium (BCIP/ NBT; Dako, Italy) substrate chromogen. Tissues were subsequently counterstained with Eosin under light microscopy.

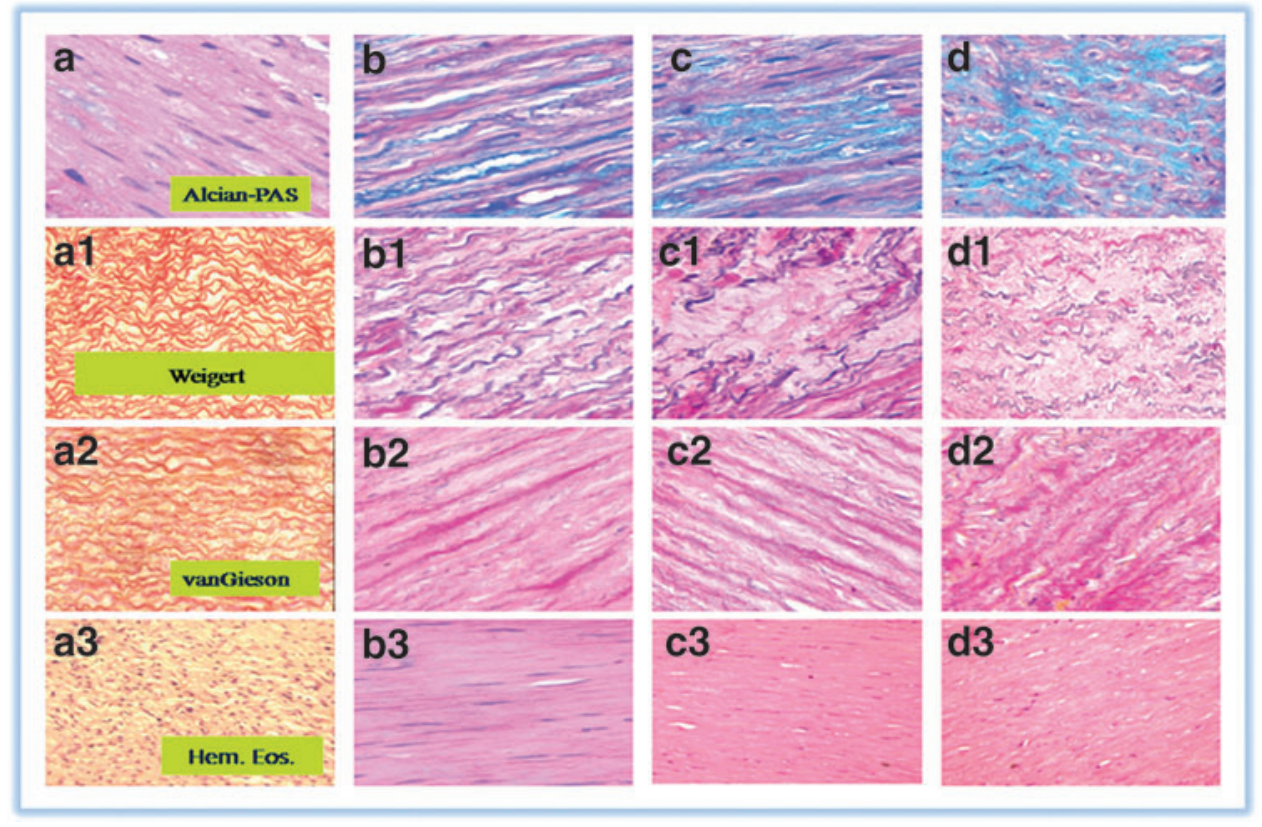

SUPPLEMENTARY FIG. 1. Control aortas and histo-pathological abnormalities in aorta tissues of S-TAA patients. Normal aorta (a, a1, a2, a3). Cystic medial changes of grade I (b), II (c) and III (d); Elastic fragmentation of grade I (b1), II (c1) and III (d1). Medial fibrosis of grade I (b2), II (c2) and III (d2). Medial necrosis of grade I (b3), II (c3) and III (d3). 


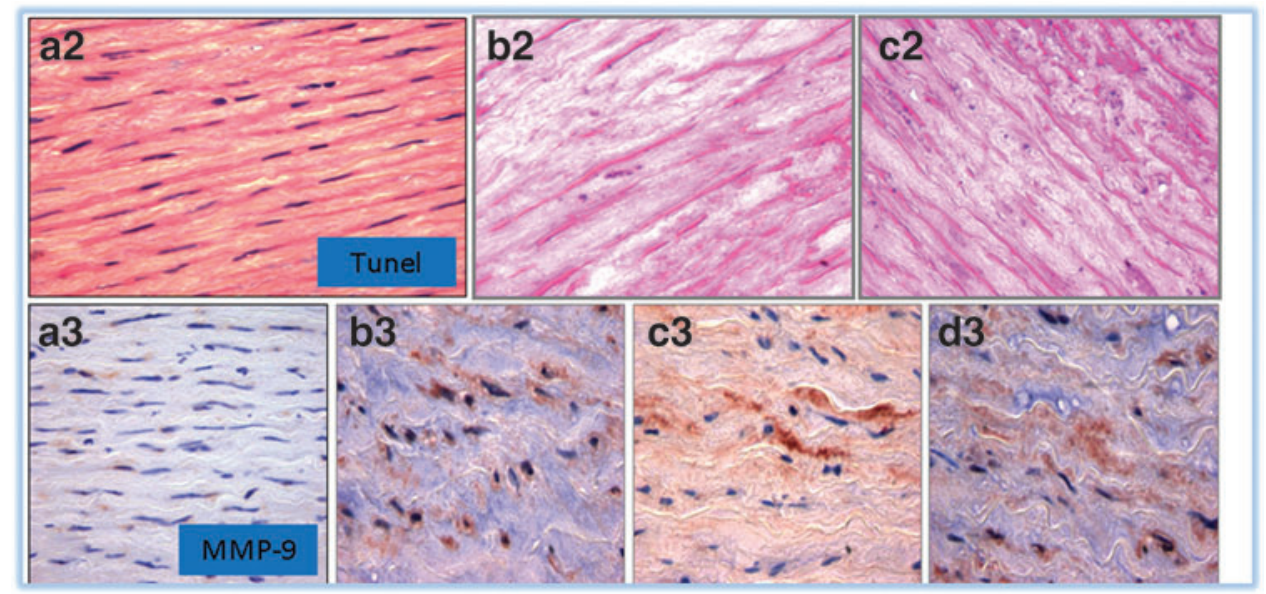

SUPPLEMENTARY FIG. 2. Medial apoptosis and MMP-9 amounts in tissue samples. In a2 and a3 images of control aorta wall conditions. In b2 and c2 images of focal and plurifocal medial apoptosis, respectively, in patient tissues. In b3 (low), c3 (moderate) and d3 (elevated) levels of MMP-9 in patient samples. 